\title{
Alternative Method for Determining the Feynman Propagator of a Non-Relativistic Quantum Mechanical Problem ${ }^{\star}$
}

\author{
Marcos MOSHINSKY $Y^{\dagger}$, Emerson SADURNI ${ }^{\dagger}$ and Adolfo DEL CAMPO \\ † Instituto de Física Universidad Nacional Autónoma de México, \\ Apartado Postal 20-364, 01000 México D.F., México \\ E-mail: moshi@fisica.unam.mx,sadurni@fisica.unam.mx \\ $\ddagger$ Departamento de Química-Física, Universidad del País Vasco, Apdo. 644, Bilbao, Spain \\ E-mail: qfbdeeca@lg.ehu.es
}

Received August 21, 2007, in final form November 13, 2007; Published online November 22, 2007

Original article is available at http://www.emis.de/journals/SIGMA/2007/110/

\begin{abstract}
A direct procedure for determining the propagator associated with a quantum mechanical problem was given by the Path Integration Procedure of Feynman. The Green function, which is the Fourier Transform with respect to the time variable of the propagator, can be derived later. In our approach, with the help of a Laplace transform, a direct way to get the energy dependent Green function is presented, and the propagator can be obtained later with an inverse Laplace transform. The method is illustrated through simple one dimensional examples and for time independent potentials, though it can be generalized to the derivation of more complicated propagators.
\end{abstract}

Key words: propagator; Green functions; harmonic oscillator

2000 Mathematics Subject Classification: 81V35; 81Q05

\section{Introduction}

It is well know that quantum mechanics acquired its final formulation in 1925-1926 through fundamental papers of Schrödinger and Heisenberg. Originally these papers appeared as two independent views of the structure of quantum mechanics, but in 1927 Schrödinger established their equivalence, and since then one or the other of the papers mentioned have been used to analyze quantum mechanical systems, depending on which method gave the most convenient way of solving the problem. Thus the existence of alternative procedures to solve a given problem can be quite fruitful in deriving solutions of it.

In the 1940's Richard Feynman, and later many others, derived a propagator for quantum mechanical problems through a path integration procedure. In contrast with the Hamiltonian emphasis in the original formulation of quantum mechanics, Feynmans approach could be referred to as Lagrangian and it emphasized the propagator $K\left(x, t, x^{\prime}, t^{\prime}\right)$ which takes the wave function $\psi\left(x^{\prime}, t^{\prime}\right)$ at the point $x^{\prime}$ and time $t^{\prime}$ to the point $x$, at time $t$, i.e.

$$
\psi(x, t)=\int K\left(x, t, x^{\prime}, t^{\prime}\right) \psi\left(x^{\prime}, t^{\prime}\right) d x^{\prime}
$$

While this propagator could be derived by the standard methods of quantum mechanics, Feynman invented a procedure by summing all time dependent paths connecting points $x, x^{\prime}$ and

${ }^{\star}$ This paper is a contribution to the Proceedings of the Seventh International Conference "Symmetry in Nonlinear Mathematical Physics" (June 24-30, 2007, Kyiv, Ukraine). The full collection is available at http://www.emis.de/journals/SIGMA/symmetry2007.html 
this became an alternative formulation of quantum mechanics whose results coincided with the older version when all of them where applicable, but also became relevant for problems that the original methods could not solve. Feynmans procedure first led to the propagator $K\left(x, t, x^{\prime}, t^{\prime}\right)$ and then by a Laplace transform to the corresponding Green function $G\left(x, x^{\prime}, E\right)$ with $E$ being the energy. We found Feynmans method for deriving the propagator, though entirely correct, somewhat cumbersome to use, and thus tried to look for alternative procedures.

As we mentioned before, in Feynmans approach the first step is deriving the propagator $K\left(x, t, x^{\prime}, t^{\prime}\right)$ and later the energy dependent Green functions $G\left(x, x^{\prime}, E\right)$. In this paper we invert the procedure, we start by deriving the $G\left(x, x^{\prime}, E\right)$ which is a simpler problem, at least in the one dimensional single particle case we will be discussing here. Once we have $G\left(x, x^{\prime}, E\right)$ the $K\left(x, t, x^{\prime}, t^{\prime}\right)$ is given by the inverse Laplace transform and can be written as

$$
K\left(x, x^{\prime}, t\right)=\frac{1}{2 \pi \hbar i} \int_{i \hbar c-\infty}^{i \hbar c+\infty} \exp (-i E t / \hbar) G\left(x, x^{\prime}, E\right) d E,
$$

where $c$ is a constant that allows the upper line $i \hbar c+E$ in the complex plane of $E$ to be above all the poles of $G\left(x, x^{\prime}, E\right)$. For compactness in the notation from now on we will take $t^{\prime}=0$ so we write $K\left(x, t, x^{\prime}, t^{\prime}\right)$ as $K\left(x, x^{\prime}, t\right)$.

The real hard part in our approach will be the determination by $(2)$ of $K\left(x, x^{\prime}, t\right)$ but this is a well defined problem in mathematics and procedures have been developed to solve it.

This is then the program we plan to follow. In Section 2 we show that for a single particle in one dimension (the initial case of our analysis) all we need to know are two independent solutions $u_{E}^{ \pm}$of the equation.

$$
\left[\frac{-\hbar^{2}}{2 m} \frac{d^{2}}{d x^{2}}+V(x)-E\right] u_{E}^{ \pm}(x, E)=0
$$

to be able to derive $G\left(x, x^{\prime}, E\right)$ in Section 3 .

We then consider in Section 4, three elementary cases, the free one dimensional particle, the corresponding one with a $\delta$ interaction at the origin $x=0$, and the harmonic oscillator. In the first two cases the integral (2) is trivial to evaluate. In the case of the harmonic oscillator the evaluation of (2) requires a more careful analysis but it can be carried out. In all three cases our final result is identical to the one presented in the book of Grosche and Steiner [1] that use Feynmans method to derive the results.

Thus we have an alternative method for deriving $K\left(x, x^{\prime}, t\right)$ but it remains to be shown that it can be applied to more particles in more dimensions and of arbitrary angular momenta and whether the analysis can be extended to relativistic as well as time dependent problems.

What ever results may be obtained in the future, it seems that our alternative approach follows more closely the standard procedures of quantum mechanics and could be useful in a simpler derivation of the propagators.

\section{The Hamiltonian of the problem and the equation for the propagator}

We start with the simplest Hamiltonian of one particle in one dimension, i.e.

$$
H=-\frac{\hbar^{2}}{2 m} \frac{d^{2}}{d x^{2}}+V(x)
$$

with thus far an arbitrary potential $V(x)$. 
From the equation (1) that defines the properties of the propagator it must satisfy the equation

$$
\left[-\frac{\hbar^{2}}{2 m} \frac{\partial^{2}}{\partial x^{2}}+V(x)-i \hbar \frac{\partial}{\partial t}\right] K\left(x, x^{\prime}, t\right)=0
$$

and besides if $t=0$ it becomes

$$
K\left(x, x^{\prime}, 0\right)=\delta\left(x-x^{\prime}\right) .
$$

We proceed now to take the Laplace transform of (3)

$$
\begin{aligned}
& \int_{0}^{\infty} \exp (-s t)\left[-\frac{\hbar^{2}}{2 m} \frac{\partial^{2}}{\partial x^{2}}+V(x)-i \hbar \frac{\partial}{\partial t}\right] K\left(x, x^{\prime}, t\right) d t \\
& \quad=-\frac{\hbar^{2}}{2 m} \frac{\partial^{2} \bar{G}\left(x, x^{\prime}, s\right)}{\partial x^{2}}+V(x) \bar{G}\left(x, x^{\prime}, s\right)-i \hbar \int_{0}^{\infty} \exp (-s t) \frac{\partial K\left(x, x^{\prime}, t^{\prime}\right)}{\partial t} d t=0,
\end{aligned}
$$

where

$$
\bar{G}\left(x, x^{\prime}, s\right) \equiv \int_{0}^{\infty} e^{-s t} K\left(x, x^{\prime}, t\right) d t .
$$

We note though that

$$
\begin{aligned}
\int_{0}^{\infty} & \exp (-s t) \frac{\partial K\left(x, x^{\prime}, t\right)}{\partial t} d t=\int_{0}^{\infty} \frac{\partial}{\partial t}\left[e^{-s t} K\left(x, x^{\prime}, t\right)\right] d t+s \int_{0}^{\infty} e^{-s t} K\left(x, x^{\prime}, t\right) d t \\
& =-\delta\left(x-x^{\prime}\right)+s \bar{G}\left(x, x^{\prime}, s\right)
\end{aligned}
$$

where we made use of (4) and (5).

With the help of (6) we see that $\bar{G}\left(x, x^{\prime}, s\right)$ satisfies

$$
\left[-\frac{\hbar^{2}}{2 m} \frac{d^{2}}{d x^{2}}+V(x)-i \hbar s\right] \bar{G}\left(x, x^{\prime}, s\right)=-i \hbar \delta\left(x-x^{\prime}\right),
$$

where we now have that the partial derivative with respect to $x$ becomes the ordinary one as there is no longer a time variable.

We integrate (7) with respect to $x$ in the interval $x^{\prime}-\epsilon \leq x \leq x^{\prime}+\epsilon$ and in the limit $\epsilon \rightarrow 0$ obtain two equations

$$
\begin{aligned}
& {\left[-\frac{\hbar^{2}}{2 m}\left(\frac{d \bar{G}}{d x}\right)_{x=x^{\prime}+0}+\frac{\hbar^{2}}{2 m}\left(\frac{d \bar{G}}{d x}\right)_{x=x^{\prime}-0}\right]=-i \hbar,} \\
& {\left[-\frac{\hbar^{2}}{2 m} \frac{d^{2}}{d x^{2}}+V(x)-i \hbar s\right] \bar{G}\left(x, x^{\prime}, s\right)=0, \quad x \neq x^{\prime} .}
\end{aligned}
$$

We proceed now to indicate how we can derive the explicit expression of $K\left(x, x^{\prime}, t\right)$ with the help of the Green function $\bar{G}\left(x, x^{\prime}, s\right)$ of the corresponding problem satisfying (8) and (9).

\section{Determination of the Green function and the inverse Laplace transform for the propagator}

Our interest is not to stop at equations (8), (9) for $\bar{G}\left(x, x^{\prime}, s\right)$ but actually to get $K\left(x, x^{\prime}, t\right)$ for which we can use the inverse Laplace transform [2] to get

$$
K\left(x, x^{\prime}, t\right)=\frac{1}{2 \pi i} \int_{c-i \infty}^{c+i \infty} \bar{G}\left(x, x^{\prime}, s\right) e^{s t} d s
$$


where the integration takes place along a line in the complex plane $s$ parallel to the imaginary axis and at a distance $c$ to it so that all singularities of $\bar{G}\left(x, x^{\prime}, s\right)$ in the $s$ plane are on the left of it.

To have a more transparent notation rather than the $s$ plane we shall consider an energy variable $E$ proportional to it through the relation

$$
E=i \hbar s \quad \text { or } \quad s=-i(E / \hbar)
$$

and define $G\left(x, x^{\prime}, E\right)$ by

$$
-i G\left(x, x^{\prime}, E\right) \equiv \bar{G}\left(x, x^{\prime},-i E / \hbar\right) .
$$

The energy Green function must be symmetric under interchange of $x$ and $x^{\prime}$, i.e.

$$
G\left(x, x^{\prime}, E\right)=G\left(x^{\prime}, x, E\right)
$$

which combines with the two equations (8), (9) to give in this notation

$$
\begin{aligned}
& {\left[\frac{d G}{d x}\right]_{x=x^{\prime}+0}-\left[\frac{d G}{d x}\right]_{x=x^{\prime}-0}=-\frac{2 m}{\hbar},} \\
& {\left[-\frac{\hbar^{2}}{2 m} \frac{d^{2}}{d x^{2}}+V(x)-E\right] G\left(x, x^{\prime}, E\right)=0 \quad \text { for } \quad x \neq x^{\prime} .}
\end{aligned}
$$

Let us first consider the case when $x<x^{\prime}$ and proceed to show that the equations (11)-(13) determine in a unique way the Green function of the problem. For this purpose we introduce with the notation $u_{E}^{ \pm}(x)$ two linearly independent solutions of the equation (13)

$$
\left[-\frac{\hbar^{2}}{2 m} \frac{d^{2}}{d x^{2}}+V(x)-E\right] u_{E}^{ \pm}(x)=0 .
$$

From this equation we see that

$$
u_{E}^{-}(x) \frac{d^{2} u_{E}^{+}(x)}{d x}-u_{E}^{+} \frac{d^{2} u_{E}^{-}(x)}{d x}=\frac{d}{d x}\left(u_{E}^{-} \frac{d u_{E}^{+}}{d x}-u_{E}^{+} \frac{d u_{E}^{-}}{d x}\right)=0 .
$$

Thus the Wronskian of the problem defined by

$$
W(E)=u_{E}^{-}(x) \frac{d u_{E}^{+}}{d x}-u_{E}^{+}(x) \frac{d u_{E}^{-}}{d x}
$$

is independent of $x$.

As $G\left(x, x^{\prime}, E\right)$ satisfies $(13)$ we can write it for $x<x^{\prime}$ as

$$
G\left(x, x^{\prime}, E\right)=F\left(x^{\prime}, E\right) u_{E}^{+}(x),
$$

choosing one of the two solutions of equation $(13)$ and $F\left(x^{\prime}, E\right)$ is as yet an undetermined function of $x^{\prime}, E$.

We see from the symmetry of $G\left(x, x^{\prime}, E\right)$ that it must satisfy the same equation (13) in $x^{\prime}$ so that from (15) we get

$$
\left[-\frac{\hbar^{2}}{2 m} \frac{d^{2}}{d x^{\prime 2}}+V\left(x^{\prime}\right)-E\right] F\left(x^{\prime}, E\right)=0
$$

and thus $F\left(x^{\prime}, E\right)$ must a be linear combination of the two independent solutions $u_{E}^{ \pm}(x)$, i.e.

$$
F\left(x^{\prime}, E\right)=a_{+}(E) u_{E}^{+}\left(x^{\prime}\right)+a_{-}(E) u_{E}^{-}\left(x^{\prime}\right)
$$


and our Green function becomes

$$
G\left(x, x^{\prime}, E\right)=\left[a_{+}(E) u_{E}^{+}\left(x^{\prime}\right)+a_{-}(E) u_{E}^{-}\left(x^{\prime}\right)\right] u_{E}^{+}(x),
$$

while for the other case, i.e. $x>x^{\prime}$, the symmetry of the Green function demands

$$
G\left(x, x^{\prime}, E\right)=\left[a_{+}(E) u_{E}^{+}(x)+a_{-}(E) u_{E}^{-}(x)\right] u_{E}^{+}\left(x^{\prime}\right) .
$$

Replacing (16) and (17) in (12) we find that the coefficient $a_{+}(E)$ vanishes and $a_{-}(E)$ satisfies

$$
a_{-}(E) W(E)=-\frac{2 m}{\hbar} .
$$

Thus from (16), (17) and (18) we get that

$$
G\left(x, x^{\prime}, E\right)=-\frac{2 m}{\hbar} W^{-1}(E) \begin{cases}u_{E}^{-}\left(x^{\prime}\right) u_{E}^{+}(x) & \text { if } x<x^{\prime} \\ u_{E}^{-}(x) u_{E}^{+}\left(x^{\prime}\right) & \text { if } x>x^{\prime}\end{cases}
$$

We thus have the explicit Green function of our problem once we can obtain two independent solutions of the equations (13).

Once $G\left(x, x^{\prime}, E\right)$ has been determined, the propagator $K\left(x, x^{\prime}, t\right)$ is given by the inverse Laplace transform (10) which in terms of the $E$ variable becomes

$$
K\left(x, x^{\prime}, t\right)=\frac{1}{2 \pi \hbar i} \int_{i \hbar c-\infty}^{i \hbar c+\infty} \exp (-i E t / \hbar) G\left(x, x^{\prime}, E\right) d E,
$$

where now the integral takes place in the $E$ plane over a line parallel to the real axis with all the poles of $G\left(x, x^{\prime}, E\right)$ below it.

We proceed to give some specific examples of application of our method.

\section{Specific examples}

\section{a) The free particle}

The potential $V(x)$ is taken as zero and so the equation (13) becomes

$$
\left[-\frac{\hbar^{2}}{2 m} \frac{d^{2}}{d x^{2}}-E\right] G\left(x, x^{\prime}, E\right)=0 .
$$

We introduce the variable $k$ through the definition

$$
E=\frac{\hbar^{2} k^{2}}{2 m}, \quad d E=\frac{\hbar^{2} k}{m} d k
$$

and thus the $u_{E}^{ \pm}(x)$ for this problem satisfy the equation

$$
\left[\frac{d^{2}}{d x}+k^{2}\right] u_{E}^{ \pm}(x)=0, \quad u_{E}^{ \pm}(x)=\exp ( \pm i k x)
$$

with the Wronskian (14) given by

$$
W(E)=2 i k .
$$

Thus from the two cases of (19) our function $G\left(x, x^{\prime}, E\right)$ is written compactly as

$$
G\left(x, x^{\prime} E\right)=\frac{i m}{\hbar k} \exp \left[i k\left|x-x^{\prime}\right|\right] .
$$


The propagator $K\left(x, x^{\prime}, t\right)$ is given by (20) in terms of $G\left(x, x^{\prime}, E\right)$ and substituting (21) in it and writing in terms of $k$ we get

$$
K\left(x, x^{\prime}, t\right)=\frac{1}{2 \pi} \int_{-\infty}^{\infty} \exp \left[i k\left|x-x^{\prime}\right|-i\left(\hbar k^{2} / 2 m\right) t\right] d k,
$$

where, as $G\left(x, x^{\prime}, E\right)$ has no singularities, the energy can be integrated over the real line $-\infty \leq E \leq \infty$ while $k$ has double the range of $E$.

The integral (22) can be determined by completing the square and we get

$$
K\left(x, x^{\prime}, t\right)=\sqrt{\frac{m}{2 \pi i \hbar t}} \exp \left[\frac{i m\left(x-x^{\prime}\right)^{2}}{2 \hbar t}\right]
$$

which has been derived also by many other methods.

\section{b) The case of the delta potential}

We wish now to discuss the effect on the Feynman propagator of a potential

$$
V(x)=Q(x)+b \delta(x),
$$

where $Q(x)$ in a continuous function of $x$ and we assume $b>0$ to avoid bound states of the $\delta$ potential.

The equation for $u_{E}^{ \pm}$becomes now

$$
[H+b \delta(x)-E] u_{E}^{ \pm}(x)=0,
$$

where

$$
H=-\frac{\hbar^{2}}{2 m} \frac{\partial^{2}}{\partial x^{2}}+Q(x) .
$$

The Green function $G\left(x, x^{\prime}, E\right)$ satisfies the equations (12), (13) which can be written as the single equation

$$
[H+b \delta(x)-E] G\left(x, x^{\prime}, E\right)=-i \hbar \delta\left(x-x^{\prime}\right)
$$

and (12) holds if we integrate (24) with respect to the variable $x$ in the integral $x^{\prime}-\epsilon \leq x \leq x^{\prime}+\epsilon$ in the limit $\epsilon \rightarrow 0$, and the one corresponding to (13) holds when $x \neq x^{\prime}$.

For $x \neq 0$ we have (24) with no delta potential and therefore $G$ can be written as

$$
G\left(x, x^{\prime}, E\right)=G_{Q}\left(x, x^{\prime}, E\right)+F\left(x, x^{\prime}, E\right), \quad x \neq 0,
$$

where $G_{Q}\left(x, x^{\prime}, E\right)$ is the Green function satisfying

$$
[H-E] G_{Q}\left(x, x^{\prime}, E\right)=-i \hbar \delta\left(x-x^{\prime}\right)
$$

while $F\left(x, x^{\prime}, E\right)$ is a solution of the corresponding homogeneous equation, i.e.

$$
[H-E] F\left(x, x^{\prime}, E\right)=0, \quad x \neq x^{\prime} .
$$

and the form of $F\left(x, x^{\prime}, E\right)$ is to be determined. The continuity of $G\left(x, x^{\prime}, E\right)$ at $x=0$ allows to write (25) for all values of $x, x^{\prime}$ and with this in mind we can replace (25) in (24) to obtain

$$
\begin{aligned}
& {[H-E-b \delta(x)]\left[G_{Q}\left(x, x^{\prime}, E\right)+F\left(x, x^{\prime}, E\right)\right]=-i \hbar \delta\left(x-x^{\prime}\right)} \\
& \quad=-i \hbar \delta\left(x-x^{\prime}\right)-b \delta(x) G_{Q}\left(0, x^{\prime}, E\right)+[H-E-b \delta(x)] F\left(x, x^{\prime}, E\right),
\end{aligned}
$$


where in the second line we have used (26). The two lines in (27) imply

$$
[H-E-b \delta(x)] F\left(x, x^{\prime}, E\right)=b \delta(x) G_{Q}\left(0, x^{\prime}, E\right)
$$

which is a version of (24) but with a source term. Therefore the solution can be readily given as

$$
F\left(x, x^{\prime}, E\right)=-\frac{i}{\hbar} \int_{-\infty}^{\infty} d x^{\prime \prime} G\left(x, x^{\prime \prime}, E\right)\left(b \delta\left(x^{\prime \prime}\right) G_{Q}\left(0, x^{\prime}, E\right)\right) .
$$

Performing the integral in the last expression and using (25) for $G$, we have

$$
F\left(x, x^{\prime}, E\right)=\gamma\left[G_{Q}(x, 0, E)+F(x, 0, E)\right] G_{Q}\left(0, x^{\prime}, E\right),
$$

where $\gamma \equiv-i b / \hbar$. To determine $F(x, 0, E)$ in the RHS of (28) we set $x^{\prime}=0$ and solve for $F$, obtaining

$$
F(x, 0, E)=\frac{\gamma G_{Q}(x, 0, E) G_{Q}(0,0, E)}{1-\gamma G_{Q}(0,0, E)} .
$$

Finally, (29) can be replaced back in (28) to get

$$
F\left(x, x^{\prime}, E\right)=\frac{\gamma G_{Q}(x, 0, E) G_{Q}\left(0, x^{\prime}, E\right)}{1-\gamma G_{Q}(0,0, E)} .
$$

With this, $G\left(x, x^{\prime}, E\right)$ is given now in terms of $G_{Q}\left(x, x^{\prime}, E\right)$ and if we make $Q=0$ we can apply it to the case of the free particle. The Green function $G_{0}\left(x, x^{\prime}, E\right)$ is given by $(21)$ and the Green function of our problem becomes

$$
\begin{aligned}
G\left(x, x^{\prime}, E\right) & =\frac{i m}{\hbar k} \exp \left[i k\left|x-x^{\prime}\right|\right]-\frac{m^{2} b}{2 \hbar^{4}} \frac{\exp \left[i k\left(|x|+\left|x^{\prime}\right|\right)\right]}{k\left(k+i \frac{m b}{\hbar^{2}}\right)} \\
& =\frac{i m}{\hbar k} \exp \left[i k\left|x-x^{\prime}\right|\right]-\frac{i m}{2 \hbar^{2}} \frac{\exp \left[i k\left(|x|+\left|x^{\prime}\right|\right)\right]}{k}+\frac{i m}{2 \hbar^{2}} \frac{\exp \left[i k\left(|x|+\left|x^{\prime}\right|\right)\right]}{k+i \frac{m b}{\hbar^{2}}} .
\end{aligned}
$$

This is the same result that appears in Grosche and Steiner [1, (6.12.4), p. 328] and accounts explicitly for the four possible cases $\pm x, \pm x^{\prime}>0$. The inverse Laplace transform of this expression can be easily evaluated since it contains integrals of the form

$$
\int_{-\infty}^{\infty} d k k^{-n} \exp \left(-C k^{2}+D k\right)
$$

where $C$ and $D$ are independent of $k$ and the integrals are either gaussians or error functions when $n=0$ or $n=1$ respectively. The propagator is then

$$
\begin{aligned}
K\left(x, x^{\prime}, t\right)= & K_{0}\left(x, x^{\prime}, t\right)+\frac{m b}{2 \hbar^{2}} \exp \left(-\frac{m b}{\hbar^{2}}\left(|x|+\left|x^{\prime}\right|+\frac{i m b^{2} t}{2 \hbar^{3}}\right)\right) \\
& \times \operatorname{erfc}\left\{\sqrt{\frac{m}{2 i \hbar t}}\left(|x|+\left|x^{\prime}\right|-\frac{i b t}{\hbar}\right)\right\}
\end{aligned}
$$

with $K_{0}\left(x, x^{\prime}, t\right)$ as in (23). This result coincides with the one that Grosche and Steiner [1, (6.12.2), p. 327] obtained by the Path Integral Methods.

Note also that if in $G$ of (25) we replace the $F\left(x, x^{\prime}, E\right)$ by (30) we also get the result of Grosche and Steiner [1, (6.12.1), p. 327]. 


\section{c) The harmonic oscillator}

The potential $V(x)$ is proportional to $x^{2}$ and thus $u_{E}^{ \pm}(x)$ satisfies the equation

$$
\left[-\frac{\hbar^{2}}{2 m} \frac{d^{2}}{d x^{2}}+\frac{1}{2} m \omega^{2} x^{2}-E\right] u_{E}^{ \pm}(x)=0,
$$

where $\omega$ is the frequency of the oscillator.

We introduce the variables

$$
z=\sqrt{\frac{2 m \omega}{\hbar}} x, \quad p=\frac{E}{\hbar \omega}-\frac{1}{2}
$$

in terms of which the equation (31) takes the form

$$
\left[\frac{d^{2}}{d z^{2}}-\frac{z^{2}}{4}+p+\frac{1}{2}\right] u_{E}^{ \pm}(x)=0
$$

Two independent solutions of (33) are given by parabolic cylinder functions [3], i.e.

$$
u_{E}^{ \pm}(x)=D_{p}( \pm z) .
$$

The Wronskians of these functions, where the derivative is taken with respect to the $x$ rather than the $z$ variable, is from (14) and (32) given by

$$
\begin{aligned}
W(E) & =\sqrt{\frac{2 m \omega}{\hbar}}\left\{D_{p}(-z)\left[\frac{d D_{p}(z)}{d z}\right]-D_{p}(z) \frac{d D_{p}(-z)}{d z}\right\} \\
& =\sqrt{\frac{2 m \omega}{\hbar}}\left\{D_{p}(-z)\left[-D_{p+1}(z)+\frac{1}{2} z D_{p}(z)\right]+D_{p}(z)\left[-D_{p+1}(-z)-\frac{1}{2} z D_{p}(-z)\right]\right\},
\end{aligned}
$$

where we made use $[3,(9.247-3)$, p. 1066] together with the fact that

$$
\left[d D_{p}(-z) / d z\right]=-\left[d D_{p}(-z) / d(-z)\right]
$$

The Wronskian is independent on $z$ so we may take any value of the latter and we choose $z=0$ to get

$$
W(E)=-\sqrt{\frac{2 m \omega}{\hbar}} 2 D_{p}(0) D_{p+1}(0) .
$$

We note from $\left[3,(9.240)\right.$, p. 1064] that we can write $D_{p}(z)$ in terms of the degenerate hypergeometric function $\Phi$ and, in particular

$$
D_{p}(0)=2^{p / 2} \frac{\sqrt{\pi}}{\Gamma\left(\frac{1-p}{2}\right)} \Phi\left(-\frac{p}{2}, \frac{1}{2}, 0\right)
$$

while from $[3,(9.210)$, p. 1058$]$ the $\Phi\left(-\frac{p}{2}, \frac{1}{2}, 0\right)=1$ so that finally

$$
W(E)=-\sqrt{\frac{2 m \omega}{\hbar}} \frac{2^{p+1} \pi}{\Gamma\left(\frac{1-p}{2}\right) \Gamma\left(-\frac{p}{2}\right)}=-\sqrt{\frac{2 m \omega}{\hbar}} \frac{\sqrt{\pi}}{\Gamma(-p)},
$$

where for the last expression in (34) we made use of the doubling formula for the $\Gamma$ function given in $[3,(8.335)$, p. 938]. 
From the general relation (19) we then obtain that the Green function of the oscillator is given by

$$
G\left(x, x^{\prime}, E\right)=\sqrt{\frac{2 m}{\pi \hbar \omega}} \Gamma(-p) D_{p}(z) D_{p}\left(-z^{\prime}\right)
$$

with $p, z$ given by (32), $z<z^{\prime}$ and $z^{\prime}$ has the same definition as $z$ but $x$ replaced by $x^{\prime}$. When we consider the case $z>z^{\prime}$ we have an expression which is similar to (35) but interchanging $z$ and $z^{\prime}$. The complete formula can be written in a compact way introducing the variables

$$
x_{>}=\max \left\{x, x^{\prime}\right\}, \quad x_{<}=\min \left\{x, x^{\prime}\right\}
$$

and thus we have

$$
G\left(x, x^{\prime}, E\right)=\sqrt{\frac{2 m}{\pi \hbar \omega}} \Gamma\left(\frac{1}{2}-\frac{E}{\hbar \omega}\right) D_{\frac{E}{\hbar \omega}-\frac{1}{2}}\left(\sqrt{\frac{2 m \omega}{\hbar}} x_{>}\right) D_{\frac{E}{\hbar \omega}-\frac{1}{2}}\left(-\sqrt{\frac{2 m \omega}{\hbar}} x_{<}\right) .
$$

The expression (36) is identical to $[1,(6.2 .37)$, p. 179] except for a factor of $\sqrt{2}$.

We want though to obtain $K\left(x, x^{\prime}, t\right)$ of $(20)$, but for this we can analyze the pole structure of (36) in order to evaluate the inverse Laplace transform of $G\left(x, x^{\prime}, E\right)$ by means of the residue theorem. The result of this procedure is the series known as the spectral decomposition of $K\left(x, x^{\prime}, t\right)$. This series can be evaluated by using an identity of Hermite polynomials in [4] and references cited therein. We carry out the analysis in the Appendix and get the final result

$$
K\left(x, x^{\prime}, t\right)=\left(\frac{m \omega}{2 \pi i \hbar \sin \omega t}\right)^{1 / 2} \exp \left\{\frac{i m \omega}{2 \hbar \sin \omega t}\left[\left(x^{\prime 2}+x^{2}\right) \cos \omega t-2 x x^{\prime}\right]\right\}
$$

which coincides with expression in [1, p. 160].

\section{Conclusion}

We will state here the full steps to get the Feynman propagator of a non-relativistic single particle one dimensional problem.

Our Hamiltonian is

$$
H=\left[-\frac{\hbar^{2}}{2 m} \frac{\partial^{2}}{\partial x^{2}}+V(x)\right]
$$

with an arbitrary potential $V(x)$.

We assume that two independent eigen-functions of energy $E$ can be found and denoted by $u_{E}^{ \pm}(x)$.

We need then to determine the Wronskian

$$
W(E)=u_{E}^{-}(x) \frac{d u_{E}^{+}(x)}{d x}-u_{E}^{+}(x) \frac{d u^{-}(x)}{d x}
$$

whose value, as we know, is independent on $x$.

Following the analysis of Section 3 we can determine the energy Green function

$$
G\left(x, x^{\prime}, E\right)=-\frac{2 m}{\hbar} W(E)^{-1} \begin{cases}u_{E}^{-}\left(x^{\prime}\right) u_{E}^{+}(x) & \text { if } x<x^{\prime}, \\ u_{E}^{-}(x) u_{E}^{+}\left(x^{\prime}\right) & \text { if } x>x^{\prime} .\end{cases}
$$

Using the Laplace transform the Feynman propagator becomes

$$
K\left(x, x^{\prime}, t\right)=\frac{1}{2 \pi \hbar i} \int_{i \hbar c-\infty}^{i \hbar c+\infty} \exp (-i E t / \hbar) G\left(x, x^{\prime}, E\right) d E .
$$




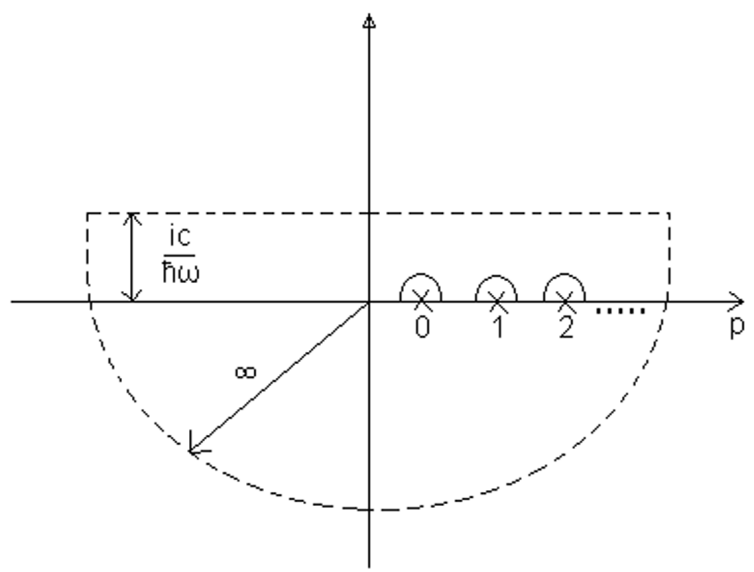

Figure 1. Integration contour for the determination of the propagator.

Once we get $u_{E}^{ \pm}(x)$ the only troublesome part of our calculation is the integral (37) as we already saw in the discussion of the examples in Section 4.

For time dependent Hamiltonians probably other techniques should be used but we have not developed them yet.

\section{A Determination of the propagator for the harmonic oscillator}

We shall proceed to express the Green function $G\left(x, x^{\prime}, E\right)$ in terms of its energy poles and residues and then apply the Laplace transform to it to get the propagator.

To achieve our objective we start with the complex variable integral

$$
\frac{\omega}{2 \pi i} \int_{C} e^{-i \hbar \omega\left(p+\frac{1}{2}\right) t} A \Gamma(-p) D_{p}(z) D_{p}(-z) d P,
$$

where

$$
p=\frac{E}{\hbar \omega}-\frac{1}{2}, \quad z=\sqrt{\frac{2 m \omega}{\hbar}} x, \quad z^{\prime}=\sqrt{\frac{2 m \omega}{\hbar}} x^{\prime}, \quad A=\sqrt{\frac{2 m}{\pi \hbar \omega}},
$$

and the contour $C$ of integration is depicted in Fig. 1.

The function $\Gamma$ is gamma while the parabolic cylinder is function $D_{p}(z)$. If we substitute $p$, $z, z^{\prime}$ of (39) in the integration (38) and replace the contour in Fig. 1 just by its upper line we obtain the integral

$$
K\left(x, x^{\prime}, t^{\prime}\right)=\frac{1}{2 \pi \hbar i} \int_{i \hbar c-\infty}^{i \hbar c+\infty} \exp (-i E t / \hbar) \sqrt{\frac{2 m}{\hbar^{3} \pi \omega}} \Gamma\left(\frac{1}{2}-\frac{E}{\hbar \omega}\right) D_{\frac{E}{\hbar \omega}-\frac{1}{2}}(z) D_{\frac{E}{\hbar \omega}-\frac{1}{2}}\left(-z^{\prime}\right) d E .
$$

We proceed now to evaluate integral (38) in terms of energy poles and residues of its integrand.

The parabolic cylinder functions $D_{p}(z), D_{p}\left(-z^{\prime}\right)$ are analytic in the full $p$ plane as shown in $[3,(2)$, p. 1066], while $\Gamma(-p)$ has poles only on the real $p$ axis with values

$$
p=n=0,1,2, \ldots
$$

Note also that the residues of $\Gamma(-p)$ at the poles given in (40) are

$$
\operatorname{Res} \Gamma(-p)=\frac{(-1)^{n}}{n !}
$$

as shown at [3, p. 933]. 
Furthermore the integral

$$
\frac{1}{2 \pi i} \int_{-\infty}^{\infty} \frac{D_{p}(z) D_{p}\left(-z^{\prime}\right)}{p-n} d p=D_{n}(z) D_{n}\left(-z^{\prime}\right)
$$

as $D_{p}(z)$ is analytic in the full $p$ plane.

The lower circle in the contour of Fig. 1 does not contribute to the integral because the term $\exp (-i \omega t p)$ vanishes when $p \rightarrow \infty$ with a negative imaginary part. As the poles marked with across in Fig. 1 are only on the real $p$ axis we can eliminate the region between the upper line and the real axis and all that remains is the residue of $\Gamma(-p)$ in the integrand $(38)$ so $K\left(x, x^{\prime}, t\right)$ takes the form

$$
K\left(x, x^{\prime}, t\right)=\omega \sqrt{\frac{2 m}{\pi \hbar \omega}} \sum_{n=0}^{\infty} \frac{(-1)^{n}}{n !} D_{n}\left(\sqrt{\frac{2 m \omega}{\hbar} x}\right) D_{n}\left(-\sqrt{\frac{2 m \omega}{\hbar}} x^{\prime}\right) e^{-i \omega\left(n+\frac{1}{2}\right) t} .
$$

The parabolic cylinder of index $n$ can be put in terms of an Hermite polynomial

$$
D_{n}(z)=2^{-\frac{n}{2}} e^{-\frac{z^{2}}{4}} H_{n}\left(\frac{z}{\sqrt{2}}\right)
$$

so the propagator can also be written as

$$
\begin{aligned}
K\left(x, x^{\prime}, t\right)= & \sqrt{\frac{2 m \omega}{\hbar \pi}} \sum_{n=0}^{\infty} \frac{e^{-i \omega(n+1 / 2) t}}{n ! 2^{n}} \\
& \times \exp \left\{-\frac{\omega m}{2 \hbar}\left(x^{2}+x^{\prime 2}\right)\right\} H_{n}\left(\sqrt{\frac{m \omega}{\hbar}} x\right) H_{n}\left(-\sqrt{\frac{m \omega}{\hbar}} x^{\prime}\right) .
\end{aligned}
$$

Using the relation [4]

$$
\sum_{n=0}^{\infty} \frac{H_{n}(z) H_{n}\left(z^{\prime}\right)}{n !} \xi^{n}=\frac{1}{\sqrt{1-4 \xi^{2}}} \exp \left\{\frac{2 \xi\left(2 \xi\left(z^{2}+z^{\prime 2}\right)-2 z z^{\prime}\right)}{4 \xi^{2}-1}\right\},
$$

we obtain

$$
\begin{aligned}
K\left(x, x^{\prime} ; t\right)= & \sqrt{\frac{2 m \omega}{\hbar \pi}} e^{-\frac{i \omega t}{2}} \exp \left\{-\frac{m \omega}{2 \hbar}\left(x^{2}+x^{\prime 2}\right)\right\} \frac{1}{\sqrt{1-e^{-i 2 \omega t}}} \\
& \times \exp \left\{\frac{m \omega}{\hbar} \frac{e^{-i \omega t}\left(e^{-i \omega t}\left(x^{2}+x^{\prime 2}\right)-2 x x^{\prime}\right)}{e^{-2 i \omega t}-1}\right\} \\
= & \sqrt{\frac{2 m \omega}{\pi \hbar}} \frac{1}{\sqrt{e^{i \omega t}-e^{-i \omega t}}} \exp \left\{\frac{m \omega}{\hbar}\left[\left(x^{2}+x^{\prime 2}\right)\left(\frac{e^{-2 i \omega t}}{e^{-2 i \omega t}-1}-\frac{1}{2}\right)-\frac{2 e^{-i \omega t} x x^{\prime}}{e^{-2 i \omega t}-1}\right]\right\} \\
= & \sqrt{\frac{m \omega}{2 i \pi \hbar \sin \omega t}} \exp \left\{\frac{i m \omega}{2 \hbar \sin (\omega t)}\left[\cos (\omega t)\left(x^{2}+x^{\prime 2}\right)-2 x x^{\prime}\right]\right\} .
\end{aligned}
$$

As equation (41) is identical to (37) and this also applies to the other corresponding equations discussed in the examples, we see that our alternative approach is equivalent to that of Feynman.

Other methods for deriving the propagator for the case of the harmonic oscillator have been proposed recently $[5,6]$.

\section{Acknowledgements}

One of the authors (A. del Campo) would like to express his thanks for the hospitality of the Instituto de Física and the support both from the Instituto de Física and of CONACYT (Project No. 40527F) for the time he spent in Mexico. This author would also like to thank the Basque Government (BFI04.479) for financial support. E. Sadurní is grateful to CONACYT and its support through Beca-Crédito 171839. M. Moshinsky is grateful to his secretary Fanny Arenas for the capture of this manuscript and the 300 she has done previously. 


\section{References}

[1] Grosche C., Steiner F., Handbook of Feynman path integrals, Springer Tracts in Modern Physics, Vol. 145, Springer, Berlin, 1998.

[2] Jaeger J.C., An introduction to Laplace transform, Methuen, London, 1965.

[3] Gradshteyn I., Ryzhik I., Table of integrals, series and products, Academic Press, 1965.

[4] Sakurai J.J., Modern quantum mechanics, Addison-Wesley, 1994.

[5] Holstein B.R., The linear potential propagator, Amer. J. Phys. 65 (1997), 414-418.

Holstein B.R., The harmonic oscillator propagator, Amer. J. Phys. 66 (1998), 583-589.

[6] Cohen S.M., Path integral for the quantum harmonic oscillator using elementary methods, Amer. J. Phys. 66 (1998), 537-540. 